Quaderni di Geografia Cahiers de Gécongaty rataphie

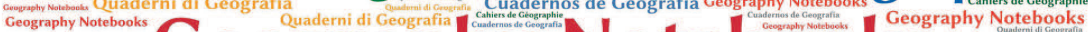
Cuadernos de Geografia Geography Notebooks $U 201000$ Cahiers de Géographi Cahiers de Géographie cahiers de Géographie Cuadernos de Geografía Cahiers de Géographie Cahiers de Géographie Cuadernos de Geografía Geography Notebooks

\author{
$4(2021)$ \\ 1 \\ Teatro di suoni. \\ Spazi acustici teatrali e territoriali
}

A cura di

Martino Mocchi, Lorena Rocca, Demis Quadri and Carlotta Sillano

EDITORIAL

Teatro di suoni per l'attaccamento ai luoghi. Uno sguardo geografico 11

Lorena Rocca

Per un teatro di suoni. Riflessioni su possibili dimensioni sonore nelle 23 creazioni site-specific di physical theatre

Demis Quadri

INTRODUCTION

Teatro di suoni. Spazi acustici teatrali e territoriali

Demis Quadri e Lorena Rocca

SPECIAL Issue

I suoni di Mantova come strumenti di interpretazione del paesaggio.

Tra turismo sostenibile ed educazione al patrimonio culturale

Valeria Pecorelli, Franca Zuccoli, Alessandra De Nicola, Enrico Squarcina

Il paesaggio sonoro campano tra contemporaneità e nuove forme

di progettualità turistica

Germana Citarella 
La narrazione spettacolarizzata del paesaggio sonoro.

Da Giuseppe Chiari a Philip K. Dick e oltre

Francesco Michi

Musica di paesaggi sonori. Enunciazione, risignificazione, comunicazione

Carlotta Sillano

Camminare per ascoltare. Partiture invisibili del territorio abitato

Elisabetta Senesi

Il paesaggio sonoro in relazione. Suono, movimento e immagini per stimolare complessità percettiva Angela Calia

Groove Fields. Understanding the Dance Floor from an Art-Based Research Perspective

Sebastian Mattbias

Il silenzio come esperienza trasformativa. L'importanza del silenzio nella meditazione e in ambito professionale

Sebastiano Caroni

Progettare il silenzio. Una lettura acustica dell'ex villaggio sanatoriale 125 Morelli a Sondalo

Martino Mocchi

Voicing One's Will. Theatre as Audio-Visual Hypotyposis of the Poetic

Michael Groneberg

Music and Clowning in Europe, 20th-21st centuries

Anna Stoll Knecht

Il paesaggio sonoro nella composizione musicale. Un percorso bibliografico

Stefano Alessandretti

\#exploreART: il labirinto di A. Pomodoro e i bambini. Un progetto di fruizione condivisa con percorsi sensoriali partecipati Alessandra De Nicola, Franca Zuccoli 


\section{OTHER EXPLORATIONS}

Il rumore lontano. Intervista a Lorena Rocca

a cura di Martino Mocchi

Re Cervo. Intervista a Antonella Astolfi

a cura di Krizia Bonaudo e Demis Quadri

Centovalli-Centoricordi. Intervista a Oliviero Giovannoni a cura di Krizia Bonaudo e Demis Quadri

Alla ricerca di un metodo: Open Space Technology 



\title{
Alla ricerca di un metodo: Open Space Technology
}

\author{
Lorena Rocca ${ }^{1}$, Martino Mocchi ${ }^{2}$ \\ ${ }^{1}$ Università degli Studi di Padova; Dipartimento DFA, SUPSI \\ ${ }^{2}$ Dipartimento ABC, Politecnico di Milano; Dipartimento DFA, SUPSI
}

DOI: https://doi.org/10.7358/gn-2021-001-roc3

\section{Open Space Technology ${ }^{1}$}

Il confronto da cui hanno preso forma alcune delle riflessioni presentate in questo numero è stato promosso attraverso uno strumento di lavoro partecipativo: l'Open Space Technology ${ }^{2}$. Si tratta di un metodo d'interazione che scommette sulla spontaneità, lasciando ai partecipanti la definizione dei temi da trattare. Harrison Owen (l'ideatore del metodo) osservò come spesso il momento più apprezzato di una conferenza fosse il coffee break. "Ciò che succede è piuttosto semplice: le persone possono scegliere i propri interlocutori e parlare di un argomento che hanno a cuore. Questa libertà consente ai partecipanti di utilizzare al meglio il loro tempo, e come conseguenza di sentirsi attivi, interessati e produttivi in quello che stanno facendo". Il principale obiettivo dell'OST non

${ }^{1}$ La presente introduzione al metodo è di Lorena Rocca, l'esito delle discussioni nel paragrafo seguente a cura di Martino Mocchi.

${ }_{2}$ Per un approfondimento del metodo: https://openspaceworld.org/cgi/itwiki.cgi; https://www.openspaceworld.com/index.htm. Si vedano inoltre le ppubblicazioni: Wiley, J. 2006. Organization Development. Jossey-Bass; Owen, H. 2008. Open Space Technology, a User's Guide. San Francisco: Berrett-Koehler. 
è infatti quello di definire con precisione chi dovrà parlare, per quanto e di che cosa, ma di strutturare un contesto di interazione che faciliti la comunicazione, rafforzi la fiducia reciproca e sprigioni la creatività dei partecipanti.

Nell'ambito del convegno Teatro di suoni, la proposta è stata quella di raccogliere le diverse suggestioni attorno a una domanda centrale: "come risuona il territorio negli spazi del teatro?". Coerentemente con il metodo, per l'attività sono state proposte quattro semplici regole: 1) chiunque partecipi è la persona giusta. Chi ha risposto all'invito è la persona giusta purché sia mossa dalla curiosità e dalla voglia di mettersi in relazione con l'altro; 2) qualsiasi cosa accada è l'unica che poteva accadere. Lasciamo perdere le aspettative e facciamoci sorprendere; 3) qualsiasi sia il momento di inizio è il momento giusto. La creatività non si manifesta a orari prestabiliti, la discussione e il confronto incominciano quando vogliamo farli incominciare; 4) quando è finita, è finita. Prendiamoci il tempo che serve, possono bastare 10 minuti o 30, l'importante è che lo riteniamo essere giusto.

Oltre alle quattro regole, vige una Legge, la Legge dei due piedi: "Se ti accorgi che non stai né imparando né contribuendo alle attività, alzati e spostati in un luogo in cui puoi essere più produttivo". Secondo questa modalità non è scortese spostarsi da un luogo all'altro, anche mentre qualcuno sta parlando, e non lo è allontanarsi da un gruppo di lavoro, se ci si vuole intrattenere con qualcuno.

Esistono due metafore che traducono possibili atteggiamenti: quella del Bombo (calabrone), un insetto rumoroso che si sposta da un luogo all'altro (le sessioni) e cosi facendo impollina e fertilizza la discussione. E quella della Farfalla, una figura silenziosa e gradevole, che si posa in un angolo creando degli spazi di distensione e di relax, migliorando il clima dei lavori. È possibile assumere entrambe le posture, è lasciata libera scelta ai partecipanti.

A partire da una breve apertura dei lavori finalizzata a condividere la domanda "come risuona il territorio negli spazi del teatro?", è stata creata un'Agenda in cui i partecipanti potevano proporre dei temi di riflessione in modo volontario. Ogni partecipante si è assunto la responsabilità di seguire la discussione in un gruppo e l'impegno di scriverne il resoconto. In questo modo sono state proposte alcune sessioni di discussione, e alla fine della giornata è stato distribuito ai partecipanti il resoconto di tutte le discussioni. Di seguito sono riportati gli esiti delle discussioni. 


\section{ESITO DELLE DISCUSSIONI}

\section{Discussione 1 - Il lago di Salmace (proponente: Michael Groenberg)}

Racconta, il Mito, di Ermafrodito figlio di Ermes e di Afrodite: "egli era bellissimo e si aggirava leggero e vacuo nei boschi della Licia. Quivi giunto incrociò un lago abitato dalla ninfa Salmace. Ella, pure bellissima, pur respinta se ne innamorò. Brigò tanto da attirare l'incauto Ermafrodito nelle acque del lago; qui lo abbracciò tanto stretto che, richiesto agli dei, i loro corpi si fusero tanto da risultare un unico corpo metà donna e metà uomo" (Ovidio, Metamorfosi, Libro Terzo).

Partendo da questo testo in cui il bellissimo ragazzo Ermafrodito che trasformato in creatura androgina si unisce fisicamente in modo soprannaturale alla ninfa Salmace, abbiamo in prima battuta intentato una trasposizione della storia considerando il lago come teatro/luogo che permetta una iniziale interpretazione del mito narrato, laddove accade la vicenda della trasformazione.

La voce diventa parte centrale del dibattito aperto da Michael (Groneberg) dove c'è un momento in cui è presente un sistema vocale uguale per tutti, uomini e donne. Antonella (Astolfi) pone però la questione in ambito fisico/evolutivo, spiegando che ci sono delle differenze sostanziali nello sviluppo delle voci umane tra maschile e femminile. La discussione verte quindi sul concetto di separazione della voce che può diventare veicolo ambientale/culturale di uno o più paesaggi (sonori?), come avviene anche nella musica e nel teatro.

Il dibattito è proseguito su come la storia/poema di Ovidio che è all'origine nel teatro può trovare un suo spazio di azione. Dopo vari scambi tra Michael (Groneberg), Sebastiano (Caroni), Martino (Mocchi), Elisabetta (Senesi) e Francesco (Michi) non si è realmente giunti a una conclusione, ma ad alcuni spunti interessanti dai quali partire per una possibile trasposizione (acustica?) del mito. Punto primo: considerare il teatro come ambito in cui il poema trova un proprio spazio, il poema a sua volta tenta un dialogo con lo spazio, e lo spazio da ultimo influenza il poema stesso. Punto secondo: considerare il concetto di "territorio" nella sua accezione ampia, includendo il "pubblico" come partecipante attivo della creazione della storia. Punto terzo: si tratta però di una storia che non è mai terminata, ma anzi continua la sua vicenda di "impersonificazione" creando nuovi teatri, e per analogia nuovi territori. 
Discussione 2 - Paesaggio, relazioni, genius loci e tradizioni musicali popolari (proponente: Germana Citarella)

La lettura del territorio necessita di vari approcci e di differenti strumenti analitici se si vuole raggiungere l'obiettivo di coglierne la complessità e, allo stesso tempo, l'unicità e le peculiarità. Le discipline geografiche, negli ultimi anni, hanno cercato di osservare il territorio non solo attraverso le sue caratteristiche fisiche, economiche e sociali o la sua struttura insediativa e l'organizzazione spaziale ma anche per il patrimonio di carattere culturale e per le componenti materiali, immateriali e semantiche che lo contraddistinguono. Potremmo affermare - parafrasando Anne Buttimer - che ogni luogo ha una storia da raccontare e che solo esplorando tale storia - intessuta di fatti, eventi, immagini, musiche - ci si può avvicinare al senso del luogo fino a coglierne l'“anima" che, secondo James Hillman, l'uomo non è più capace di riconoscere perché vittima di un fenomeno chiamato "frastuono psichico": i suoni sono così forti che le orecchie si sono intorpidite.

Molti luoghi, infatti, hanno subito la cancellazione del carattere originario a causa della genericità e della sovrabbondanza d'informazioni che, se da un lato competono tra loro per richiamare l'attenzione, dall'altro impediscono la decrittazione della realtà territoriale. Fortunatamente, nonostante la grande trasformazione indotta dalla modernità, sembra persistere nei territori qualcosa della loro interiorità ma per rintracciarla è necessario prendere coscienza di cosa contenevano, tenevano dentro, da cosa fossero in-babited.

A tal proposito, il gruppo ha riflettuto sul ruolo della musica e delle tradizioni musicali popolari come componenti rappresentative della relazione tra lo spazio e la capacità dell'uomo di esprimersi su di esso attraverso una "ibridazione complessa" composta da affetti, azioni, sensazioni, memorie e suoni. In particolare ci si è soffermati sulla Tammurriata caratteristica del mondo contadino della zona vesuviana e di quella dei Monti Lattari. Il ball'ncopp 'o tammurro è in grado non solo di arricchire la percezione del luogo in cui si è immersi in quel determinato momento, ma anche di proporre una geografia dell'emozione attraverso l'evocazione di un paesaggio della memoria che non vuole essere una "museificazione stagnante" fine a sé stessa, quanto una forma di partecipazione di tutti gli attori locali, al fine di determinare il rafforzamento e la ricomposizione di quell'identità culturale che nel tempo naturalmente si sfalda per i continui stimoli esterni ricevuti. 
Pertanto, il paesaggio sonoro generato dalle tradizioni musicali popolari può assumere un ruolo centrale nella rivitalizzazione dei territori soltanto se riflette un progetto sostenibile e condiviso anche dalla $\mathrm{co}^{-}$ munità, che ha il compito di acquisire una maggiore conoscenza della propria realtà geografica, al fine di migliorare la gestione delle risorse.

In definitiva, il paesaggio sonoro può rappresentare: a) un importante strumento cognitivo, in quanto diviene una cassa di risonanza delle specificità territoriali - storiche, economiche, sociali e culturali; b) una preziosa fonte di informazione che assume un ruolo fondamentale nel processo di "percezione collettiva" delle realtà locali. Tale ruolo diviene ancora più significativo se il suono è l'espressione degli attori locali poiché si trasforma in uno strumento endogeno di autorappresentazione identitaria e assume importanza culturale ed economica a livello locale.

\section{Discussione 3 - Paesaggi sonori, linguaggi artistici e responsabilità (proponen- te: Carlotta Sillano)}

Il lavoro artistico legato al paesaggio sonoro corrisponde spesso a un invito da parte dell'artista ad ascoltare i suoni del mondo, o di una porzione di esso. Questo invito però non è neutrale, contiene sempre un'azione, una scelta, una rielaborazione, un filtro. Paradigmatico è l'esempio di 4’33” di John Cage, il quale sembra deporre le armi di fronte ai suoni accidentali che accadono attorno a sé, ma che in realtà attiva in modo deliberato un ascolto nuovo: è il taglio nella tela, il varco nella rete. Quali sono le responsabilità, allora, e cosa vuol dire agire in modo etico nel relazionarsi ai temi del paesaggio sonoro?

Prendendo spunto dall'esperienza diretta di alcuni partecipanti alla tavola rotonda si è discusso dell'etica professionale di chi lavora con il paesaggio sonoro, rielaborandolo, utilizzandolo come materiale plasmabile, rappresentandolo. L'artista attinge da una langue inesplorata e la rende parola applicando inevitabilmente una selezione e un'interpretazione. L'obiettivo di queste pratiche è spesso una sensibilizzazione rispetto all'ascolto e alla conoscenza dei suoni del mondo. Un'operazione che può però risultare infruttuosa seppur mossa da nobili intenti. Si è così contrapposto le attività di divulgazione, gli inviti all'ascolto, le espressioni di attivismo alle pratiche di progettazione degli spazi e del suono stesso. Durante il dibattito ci si è chiesti se il modo migliore per contribuire a una diversa e più attenta percezione acustica del mondo risieda al livello progettuale e abbia a che fare con il disegno e la costruzione di spazi 
capaci di ospitare suoni migliori.

La discussione ha poi affrontato il rapporto tra arte e società. L'approccio artistico ai temi del paesaggio sonoro riflette la multidisciplinarietà dell'ambito di intervento. Difficilmente l'artista che si interfaccia con questi materiali e temi può prendere le distanze dal valore territoriale e sociale degli stessi. L'arte è forse sempre politica, poichè ha a che fare con la polis o, quantomeno, nel caso dei paesaggi sonori, con il paesaggio (reale o immaginario) che li ha generati e all'interno del quale questi hanno un ruolo semiotico, informativo, politico. Può allora il demiurgo dei suoni del mondo evitare di immergersi nelle dinamiche mondane o deve necessariamente averci a che fare? Dal dibattito è emerso come la sua opera, talvolta in modo involontario, sia forse più simile al canto dell'ape regina, che controlla e utilizza le frequenze per determinare il futuro della propria comunità.

Discussione 4 - Geografia emozionale: movimento, immagine, suono (proponente: Angela Calia)

Il tema proposto parte da un'esperienza concreta di lavoro artistico basato su uno stretto rapporto fra luogo, movimento, immagine e suono che ha avuto come obiettivo la realizzazione di cortometraggi di video danza sulla narrazione del territorio. Questo progetto, chiamato "Corpi Narranti", è nato in Italia, in provincia di Bari (Altamura) e ha come obiettivo l'esplorazione e l'applicazione di nuovi territori.

Tutto nasce dall'idea di voler conoscere e approfondire su più livelli il rapporto esistente fra il luogo, partendo dalla definizione di spazio circoscritto, e il corpo umano che a sua volta può essere inteso come "spazio circoscritto" e considerando l'idea di mappa territoriale in relazione all'idea di mappa del corpo umano.

Questa ricerca prende spunto da diversi studi nell'ambito della geografia emozionale, ma anche della geografia tradizionale, soprattutto in relazione alle carte geografiche e alle modalità con cui venivano realizzate quando ancora non era possibile utilizzare strumenti di misurazione e calcolo efficaci. Di fatto era proprio il corpo umano a essere preso come base di osservazione e di modello di realizzazione delle carte, poiché l'anatomia era una materia conosciuta e praticabile. In particolare era il corpo delle donne a essere usato maggiormente, tema sul quale si aprono altri spunti della ricerca.

Uno dei punti trattati durante il dialogo è quello della relazione fra il 
luogo fisico e il luogo del corpo: la memoria. Abbiamo parlato della trasmissione di memorie fra un corpo e l'altro, di generazione in generazione, abbiamo parlato degli aspetti emotivi e traumatici di certe esperienze che lasciano il segno nella vita delle persone, non solo a livello di bagaglio dei saperi e dei ricordi, ma anche a livello cellulare, come dimostrano le più recenti ricerche di neuroscienze ed epigenetica.

Da qui abbiamo riflettuto su lavori, fatti o possibili, relativi alla memoria dei luoghi e su come i territori, così come il corpo umano, possano essere protagonisti di processi di guarigione e trasformazione proprio attraverso percorsi creativi che permettano di sanare ferite storiche di un determinato luogo.

Come arriva il suono in questo percorso? Attraverso la consapevolezza che un processo di ricerca, consapevolezza e creazione artistica può contribuire fortemente a modificare la realtà di un luogo rendendolo energeticamente diverso e aprendo le strade a una nuova vita, consapevole ma non stantia nella sua "ferita".

Gli esempi e gli apporti relativi a questo tema sono stati molti: dal punto di vista della pedagogia si è parlato della necessità di ripensare il lavoro pedagogico in funzione di una maggiore integrazione delle arti e del rapporto fra teoria e pratica; dal punto di vista artistico sono emersi esempi concernenti l'arte contemporanea e esperimenti in questa direzione; dal punto di vista della geografia e dell'antropologia si è passati dalle pratiche sciamaniche alle più recenti ricerche universitarie dove argomenti importanti, un tempo relegati solo alle pratiche di esoterismo, diventano oggi oggetto di studio e ricerca attiva.

L'idea finale è che nell'affrontare un discorso sul territorio, soprattutto in direzione di una narrazione artistica ma anche documentaristica, non si possa prescindere né separare nettamente gli aspetti logico-razionali della costruzione e della vita di uno spazio da quelli emozionali e della memoria delle persone. È pertanto necessario sviluppare un approccio integrato sia nel trattare gli argomenti sia nel lavoro creativo.

Il suono e la musica, in questo senso, intesi come trasmissione orale e vocale di storie, memorie, fantasie, e come registrazione, creazione e riproduzione di ciò che accade nel luogo, diventano dei possibili strumenti creativi per generare connessioni, sviluppare cultura, fare integrazione. Dal punto di vista formativo, non dovrebbero più essere considerati solo come materie elitarie, dedicate ai talenti, ma come materie di formazione di base, utili allo sviluppo della psiche e delle risorse cognitive dell'individuo, nonché dello sviluppo relazionale e umano in senso ampio. 
Discussione 5 - Il rapporto tra spazi sferici e lineari (proponente: Martino Mocchi)

La discussione parte da uno spunto introdotto da Martino Mocchi sulla relazione tra spazi sferici e spazi "lineari". Coerentemente con le posizioni del filosofo Peter Sloterdjik, l'immagine della sfera rappresenta un riferimento indispensabile per la nascita e per la crescita dell'individuo. Veniamo fecondati a partire da un ovulo che si sviluppa nella placenta, siamo riconoscibili attraverso il volto, vediamo attraverso l'occhio, identifichiamo il sentimento con il cuore, ci nutriamo attraverso il seno ecc. Una sequenza di sfere corporee che fa da presupposto per lo sviluppo di sfere affettive più complesse - madre-bambino, famiglia, gruppo di amici ecc.

Tale "originarietà" della sfera si riflette in una maggiore caratterizzazione emozionale-emotiva degli spazi sferici rispetto a quelli lineari, in una loro maggiore portata fondativa rispetto ai modi del vivere sociale. Nonostante questo, l'organizzazione degli spazi collettivi dell'uomo (la città) si fonda prevalentemente su una dimensione lineare-prospettica che, sebbene giustificabile in senso funzionale, potrebbe comportare un indebolimento a livello simbolico e culturale dei legami tra l'individuo e l'ambiente.

Così esposta, la contrapposizione sfera/linea sintetizza in un'immagine geometrica alcune differenze riconducibili alla contrapposizione percettiva tra udito e vista. In molti casi ciò è confermato dall'utilizzo del suono per conferire un senso mistico e di coinvolgimento agli spazi sferici. Anche a livello fisico, lo sviluppo del suono procede secondo onde sferiche, mentre l'osservazione visiva si costruisce all'interno di una prospettiva lineare.

A seguito della presentazione del tema, l'avvio della discussione si lega a dei riferimenti emersi tra i partecipanti, come il concetto di "musica delle sfere” (Pitagora, Hildegard von Bingen, Keplero), la forma circolare della città medievale, alcune esperienze artistico-architettoniche del secolo scorso (le cupole geodetiche di Buckminster Fuller o il padiglione Philips di Le Corbusier-Xenakis).

La riflessione pone delle possibili critiche agli argomenti presentati, basate sul fatto che in alcuni casi la costruzione di spazi sferici (o almeno la disposizione circolare del gruppo attorno a un centro) è conseguente alla percezione di un pericolo, segnalando in qualche modo la necessità di prendere le distanze dal centro, più che essere conseguente alla ricerca di uno spazio intimo e coinvolgente. Inoltre, vengono evidenziati 
alcuni studi sulla percezione, dai quali emerge il carattere non-lineare della vista, facendo cadere la contrapposizione "geometrica" tra sfericità dell'udito e linearità del visivo.

Viene quindi introdotta un'altra riflessione, sull'importanza di estendere la ricerca al rapporto tra le forme geometriche e la loro connotazione morale e culturale. Una dimensione resa particolarmente attuale dalla diffusione dello "schermo" come nuovo centro percettivo e comunicativo. Un rettangolo bidimensionale che tende ad assorbire tutte le componenti simboliche e relazionali precedentemente associate alla moltitudine delle figure e alle varie dimensioni multisensoriali.

Dopo un interessante confronto attorno a questi temi, il gruppo concorda sul fatto che gli spazi sferici esprimono delle caratteristiche particolari, legate alla possibilità di: rappresentare spazi metaforici; attivare delle forme di partecipazione e di percezione differenti rispetto a quelle usuali; favorire una gerarchia differente tra i membri della comunità che li abita.

La domanda relativa a come riproporre attraverso le forme dell'architettura contemporanea i tratti simbolici della sfera viene riconosciuta quindi come un tema di interesse, che si ritiene potrà rappresentare un interrogativo per la ricerca futura. 
